American J. of Engineering and Applied Sciences 1 (2): 118-120, 2008

ISSN 1941-7020

(C) 2008 Science Publications

\title{
Pitch Angle Control of Variable Speed Wind Turbine
}

\author{
Yousif El-Tous \\ Faculty of Engineering Technology, Al-Balqa Applied University, Jordan
}

\begin{abstract}
The aim of this study is to design a simple controller to maximize the extracted energy of wind turbines. In this study the pitch angle control of variable speed wind turbine is investigated. In particular, it concentrates on the extraction of maximum available energy, reduction of torque and output power variations, which gives stresses in the gearbox and mechanical structure. The control concentrates on separate wind speed internals as well as on whole wind speed region. It is found that the control structures varies substantially between the wind speed regions. Two different control systems are compared. The results show that pitch actuator with three levels of pitching speed have better response.
\end{abstract}

Key words: Wind turbines, Pitch angle, Pitching speed, Control technology.

\section{INTRODUCTION}

The global market for wind power has been expanding faster than any other sources of renewable energy. The world wind power capacity has been duplicated around twelve times from just 4,800 MW in 1995 to reach over than 59,000 MW at the end of 2005. Wind power is now established as an energy source in over 50 countries around the world. Those with the highest totals in 2005 were Germany (18,428 MW), Spain (10,027 MW), the USA (9,149 MW), India (4,430 MW) and Denmark (3,122 MW). A number of other countries, including Italy, the UK, the Netherlands, China, Japan and Portugal, have reached the $1,000 \mathrm{MW}$ of wind power capacity ${ }^{[1]}$. Denmark is planning for wind power to cover $50 \%$ of the electricity consumption by $2025^{[2]}$.

Although the control of variable speed pitchcontrolled is an important area and obviously routines have been developed by manufacturers, there is not much to be found in the generally available literature. The steady-state operating conditions are well described by $^{[3]}$. In $\mathrm{Mika}^{[4]}$ suggestions for controllers for some wind speed regions can be found, however, no solutions of how to switch between these controllers were presented. Moreover, in ${ }^{[4]}$ the suggested pitch controller was of a very active type, that could lead to wear of the mechanical parts, which, of course, is a drawback.

Automatic control is essential for efficient and reliable operation of wind power turbines and is an interesting, challenging research topic. Nowadays, variable speed wind turbines are becoming more common than constant speed turbines.

This is mainly due to a better power quality impact, reduction of stresses in the turbine and the reduction of the weight and cost of the main components. The traditional fixed-speed turbines are stall regulated while the new, variable-speed turbines are pitch-regulated. The aerodynamic efficiency $\operatorname{Cp}(\lambda, \beta)$ is a function of the tip-speed ratio and the pitch angle $\beta$. Given a pitch angle, the efficiency coefficient $\mathrm{Cp}$ has a maximum for a certain tip-speed ratio, $\lambda$. It is thus obvious that to maximize the efficiency of the turbine, we should be able to vary its rotational speed to follow the wind speed. The fluctuating nature of the wind makes the variable speed turbine a nontrivial object to control. The objectives is to achieve high efficiency and at the same time have a smooth power output. The control variables are the electro dynamical torque and the pitch angle.

\section{MATERIALS AND METHODS}

Blade pitch control is primarily used to limit the aerodynamic power above rated wind speed in order to keep the turbine shaft torque within its design limits. The inertia of the blades turned by the drive is large and the pitch actuator has thus limited capabilities. Its dynamics are non-linear with saturation limits on pitch angle (usually from $-3^{\circ}-90^{\circ}$ ) and pitching speed rate (around $8^{\circ}-10^{\circ} \mathrm{sec}^{-1}$ ). In this study, two pitch angle control structures are evaluated.

The current study used pitch actuator as integrator with saturation. The model is developed using MATLAB Simulink toolbox. This actuator is modeled in closed loop with saturation of the pitch angle and a pitch rate limitation as shown in Fig. 1. In this closed loop configuration with integrator, its gives similar result as a first order transfer function but with 
limitation of the pitch rate. Also while the $\beta_{\text {ref }}$ is on the lower limit, the integrator is prevented from growing indefinitely or winding up.

This actuator is modeled in closed loop with saturation of the pitch angle and a pitch rate limitation. In this closed loop configuration with integrator, its gives similar result as a first order transfer function but with limitation of the pitch rate. Also while the $\beta_{\text {ref }}$ is on the lower limit, the integrator is prevented from growing indefinitely or winding up.

The second method uses pitch actuator with three levels of pitching speed. This actuator is modeled as a three pitching speed actuator. It can perform the pitching only with one fixed speed in both directions or it can remain at a constant angle. So if the signal error is greater than the trigger level, a pitch acting with a constant speed is ordered until then error signal become lower than the trigger value. When this happens the pitching stops and the angle remains in one position. The error signal comes from the controller and is usually based on the power error. The block scheme is shown in Fig. 2. This approach of actuator is slightly different than the previous one since the information for input is based on error not in a certain value.

The pitching is only active when the error level is outside the dead zone values, otherwise the pitching mechanism remain at rest. This behavior is better from a practical point of view because it needs much less pitch activity and is more "structure friendly" due to that pitch actuator will be at standstill for longer period of time. So the pitching is not performed constantly like in the first pitch actuator proposal.

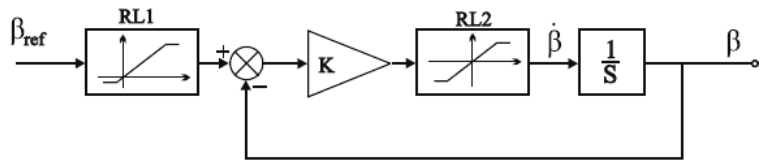

Fig. 1: Pitch actuator control of wind turbine Where:

RL1 $=$ The pitch angle limitation,

RL2 $=$ The pitching speed limitation

$\mathrm{K}=$ The softness coefficient when the limit is approaching

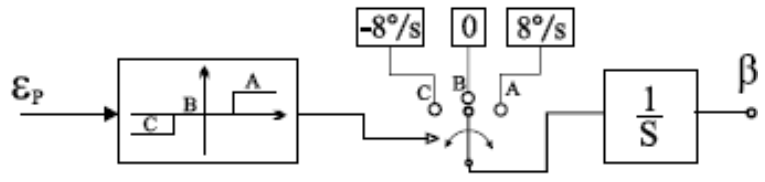

Fig. 2: Pitch actuator (second method)

\section{RESULTS AND DISCUSSION}

In Fig. 3, step responses for two different ramping speeds and two different $\mathrm{K}$ coefficients are shown for the block shown in Fig. 1. The solid line represents the reference value and the thick dashed line the used pitch angle limitation.

While the response of the block shown in Fig. 2 is shown as in Fig. 4.

The response of the controller during the time is shown as in Fig. 5 of both methods.

As can be seen from the previous figures, the controller shows a good capability of giving the right angle demand during the time at different $\mathrm{K}$ coefficients

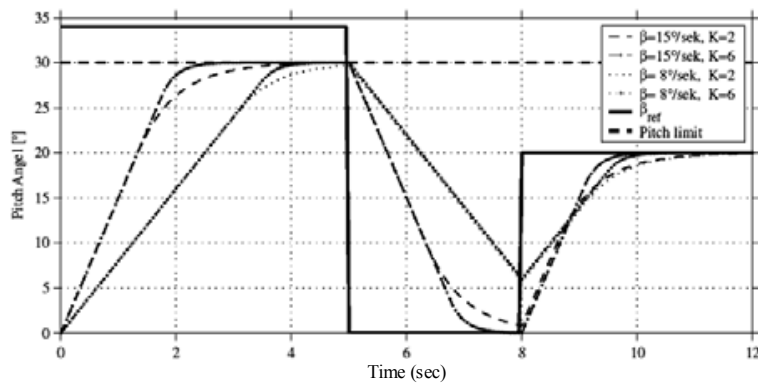

Fig. 3: Responses of pitch angel demand

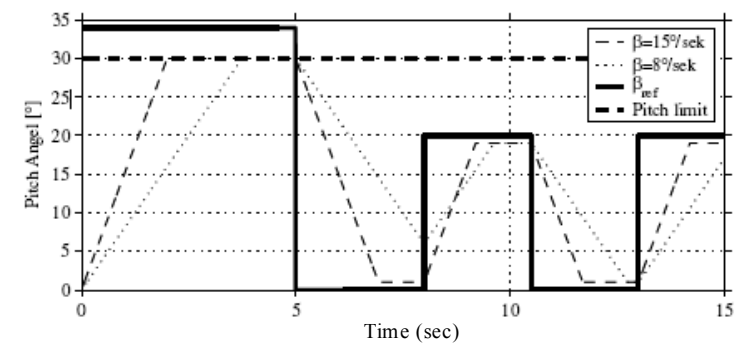

Fig. 4: Step response of the second method
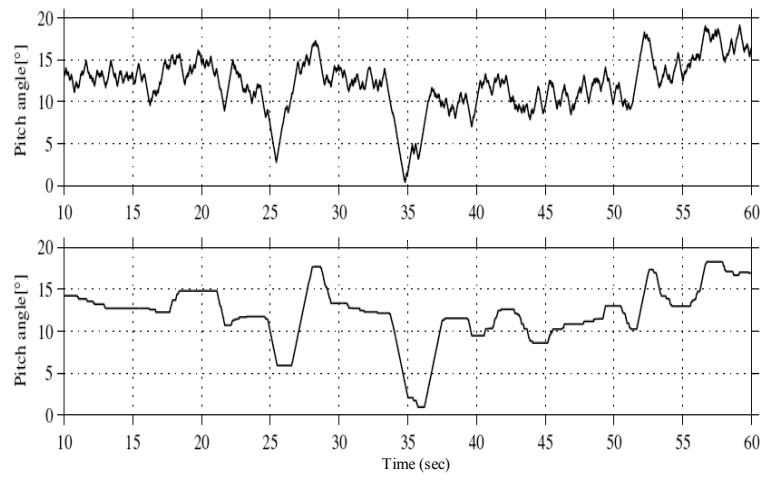

Fig. 5: Comparison of two pitch actuators activity 
in an easy way. In the Fig. 5, in the upper diagram a pitch activity with the version of actuator using integration and the lower one shows the method of using three levels of speed is shown. By adjusting the dead zone we can make a more 'sharp' or 'at' behavior and it is insensitive from a controller gain tuning point of view. In this simulation the controller parameters in both cases were tuned to get the same power deviation and make the both pitch actuators structures comparable. The first approach is very sensitive to controller gain tuning and hence the activity of pitch is continuous, as can be observed from the figure, which will wear out the mechanism unnecessarily.

The three speed level actuator (second method) seems to be a better choice. The main disadvantage of the first version actuator is that we cannot predict which pitch angle we will needed at that time when the actuator has reached command value. At this time the wind condition may have changed and then we will need another setting. The second version compare 'on line' the proper criterion and then decide, if to increase or decrease the pitch angle, without predicting the future angle like in the first version.

\section{CONCLUSION}

The complexity of the variable speed system leads to increased cost and reduced reliability due to the use of power electronics and a more complicated control.
But as a result of using a more advanced control, reduced mechanical stresses on the shaft as well as on the structure of the turbine as well as a better power quality can be achieved. A variable rotor speed turbine allow us to partially store energy from wind gusts in the rotating mass of the turbine rotor instead of transmitting them through the drive train. It is shown that in the low wind speed a controller implementation using a proper tuning a reduction of the mechanical stresses can be achieved without loosing a significant amount of energy (usually by using a slow speed controller).

\section{REFERENCES}

1. Shata AS, Hanitsch R, 2006. Evaluation of wind energy potential and electricity generation on the coast of Mediterranean Sea in Egypt. Renewable Energy; 31(8): 1183-1202.

2. Ramírez-Rosado IJ, García-Garrido Eduardo, Fernández-Jiménez L, Zorzano-Santamaría PJ, Monteiro C, Miranda V, 2008. Promotion of new wind farms based on a decision support system. Renewable Energy; 33(4): 558-566.

3. Bossanyi, E., 2001. The design of closed looped controllers for wind turbines. Wind Energy; 3(3), 149-163.

4. Mika, R., 2003. Torque and Speed Control of a Pitch Regulated Wind Turbine. M.Sc. Thesis, Chalmers University of Technology, Goteborg, Sweden. 